\title{
ARTICLE
}

\section{Conversion of hepatoma cells to hepatocyte-like cells by defined hepatocyte nuclear factors}

\author{
Zhuo Cheng ${ }^{1}$, Zhiying He $\mathbb{D}^{2}$, Yongchao $\mathrm{Cai}^{2}$, Cheng Zhang ${ }^{1}$, Gongbo Fu ${ }^{1}$, Hengyu $\mathrm{Li}^{1}$, Wen Sun ${ }^{1}$, Changcheng Liu ${ }^{2}$, Xiuliang Cui ${ }^{1}$, \\ Beifang Ning ${ }^{3}$, Daimin Xiang ${ }^{1}$, Tengfei Zhou ${ }^{1}$, Xiaofeng $\mathrm{Li}^{1}$, Weifen Xie ${ }^{3}$, Hongyang Wang ${ }^{1,4}$ and Jin Ding ${ }^{1,4}$
}

Normal cells become cancer cells after a malignant transformation, but whether cancer cells can be reversed to normal status remains elusive. Here, we report that the combination of hepatocyte nuclear factor 1A (HNF1A), HNF4A and forkhead box protein A3 (FOXA3) synergistically reprograms hepatocellular carcinoma (HCC) cells to hepatocyte-like cells (reprogrammed hepatocytes, rHeps). Our results show that rHeps lose the malignant phenotypes of cancer cells and retrieve hepatocyte-specific characteristics including hepatocyte-like morphology; global expression pattern of genes and specific biomarkers of hepatocytes; and the unique hepatic functions of albumin (ALB) secretion, glycogen synthesis, low-density lipoprotein (LDL) uptake, urea production, cytochrome P450 enzymes induction and drug metabolism. Intratumoral injection of these three factors efficiently shrank patientderived tumor xenografts and reprogrammed HCC cells in vivo. Most importantly, transplantation of rHeps in the liver of fumarylacetoacetate hydrolase-deficient $\left(\mathrm{Fah}^{-1-}\right)$ mice led to the reconstruction of hepatic lobules and the restoration of hepatic function. Mechanistically, exogenous expression of HNF1A, HNF4A and FOXA3 in HCC cells initiated the endogenous expression of numerous hepatocyte nuclear factors, which promoted the conversion of HCC cells to hepatocyte-like cells. Collectively, our results indicate the successful conversion of hepatoma cells to hepatocyte-like cells, not only extending our current knowledge of cell reprogramming but also providing a route towards a novel therapeutic strategy for cancer.

Cell Research (2019) 29:124-135; https://doi.org/10.1038/s41422-018-0111-x

\section{INTRODUCTION}

It had been accepted for a long time that embryonic stem cells (ESs) could develop into all types of differentiated cell lineages and this was thought to be irreversible. By developing somatic cell nuclear transfer (SCNT) in 1962, Gurdon et al. ${ }^{1}$ successfully reprogrammed differentiated somatic cells into pluripotent cells. Blau and colleagues ${ }^{2}$ were able to reverse somatic cells to the pluripotent status using in vitro cell fusion in 1983. Subsequently in 2006, Yamanaka and colleagues ${ }^{3}$ made the striking discovery that somatic cells could be converted into induced pluripotent cells (iPS) by introducing Oct4, Sox2, KIf4 and c-Myc, thus providing a relatively striaghtforward technique for obtaining patient-specific pluripotent stem cells, which offer enormous clinical significance. Inspired by these pluripotent reprogramming processes, Feng and co-workers ${ }^{4}$ successfully converted fibroblasts into macrophage-like cells by forced expression of C/EBPa or C/EBP $\beta$ with PU.1 in 2008. This realized the direct conversion of terminally differentiated lineages with different germ layers of origin, and became termed trans-differentiation or lineage reprogramming.

Mouse fibroblasts have been induced to form functional hepatocyte-like cells (iHeps) by expressing hepatocyte specific nuclear factors. ${ }^{5,6}$ It also proved possible to generate mouse- and human-induced neuronal cells by introducing neuron-specific transcription factors into fibroblasts although different transcription factors had to be used for different species. ${ }^{7,8}$ Consistently, Huang et al. $^{9}$ induced human fibroblasts to form functional hepatocyte-like cells (hiHeps) using HNF1A, HNF4A and FOXA3, a different set of factors to those used in mice. Later, an increasing number of terminally differentiated cell types from both mice and humans were induced to develop into other cell lineages using distinct transcription factors. ${ }^{10}$ Together, these findings indicate that cell reprogramming provides a viable approach for establishing different disease models and even therapeutic strategies.

Based on these achievements, we wondered whether cancer cells could be converted into normal cells using similar approaches and so fulfil a long existing challenge. Since forced expression of HNF1A, HNF4A and FOXA3 induced human fibroblasts to form functional hepatocyte-like cells, we tested whether these three factors could lead hepatocellular carcinoma (HCC) cells to revert into hepatocytes. In contrast to HCC cells, hepatocytes exhibit a particular gene expression profile and possess unique functions, including albumin (ALB) secretion, glycogen synthesis, low-density lipoprotein (LDL) uptake as well as the mechanisms for metabolic control and detoxification. ${ }^{11}$ More importantly, transplantation of hepatocytes or induced/functional hepatocyte-like cells into the liver of fumarylacetoacetate hydrolase-deficient mice $\left(\mathrm{Fah}^{-/-}\right.$) can reconstruct hepatic lobules

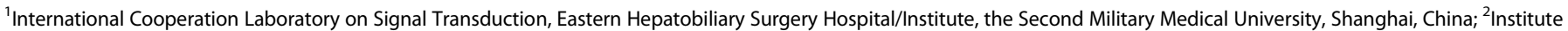

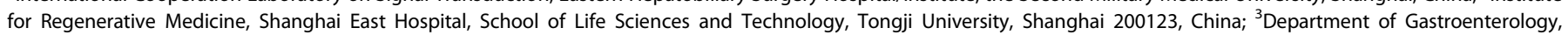
Changzheng Hospital, the Second Military Medical University, Shanghai, China and ${ }^{4}$ National Center for Liver Cancer, Shanghai, China

Correspondence: Hongyang Wang (hywangk@vip.sina.com) or Jin Ding (dingjin1103@163.com)

These authors contributed equally: Zhuo Cheng, Zhiying He, Yongchao Cai, Cheng Zhang.

Received: 11 April 2018 Accepted: 22 October 2018

Published online: 18 December 2018 
in liver that exert hepatic functions. ${ }^{12-14}$ In this study, we have investigated whether HNF1A, HNF4A and FOXA3-mediated reprogramming can convert HCC cells to hepatocyte-like cells with unique hepatic characteristics with the aim of investigating the underlying mechanism.

\section{RESULTS}

HNF1A, HNF4A and FOXA3 synergistically induce HCC cell conversion to rHeps

The liver cancer cell lines HCCLM3 and Huh-7, were selected to test whether the defined factors, HNF1A, HNF4A and FOXA3, could reprogram HCC cells to hepatocyte-like cells (Fig. 1a; Supplementary information, Fig. S1). As adenovirus shows hepatotropism, we used it as a vector to introduce HNF1A, HNF4A and FOXA3 (Adenovirus-3Factors; Ad-3F) into HCC cells to achieve their overexpression (Supplementary information, Fig. S2). Strikingly, the combination of HNF1A, HNF4A and FOXA3 exhibited synergistic effects on hepatocyte-specific gene induction in HCCLM3 and Huh-7 cells (Fig. 1b; Supplementary information, Fig. S3a). As anticipated, the levels of mature hepatocyte specific gene expression were gradually increased during the induction process, suggesting that cell fate conversion should be progressively coordinated (Fig. 1c; Supplementary information, Fig. S3b). Almost $100 \%$ of the HCC cells were infected by adenovirus, and the induced cells exhibited substantial morphological changes 7 days after Ad-3F induction (Fig. 1d). Compared with Ad-GFP-treated control cells, Ad-3Finduced cells exhibited hepatocyte-like morphology with a flat and sometimes binuclear phenotype leading us to term them, reprogrammed hepatocytes (rHeps). Nuclear segmentation in control cells was evident using scanning electron microscopy, whereas the nuclei of rHeps were well-organized (Supplementary information, Fig. S4). Moreover, rHeps displayed a dramatic reduction of AFP and a notable induction of ALB. An ELISA assay also showed that rHeps have a robust capability for secreting the plasma protein ALB at a level resembling that of primary human hepatocytes (Fig. 1e). Microarray analysis indicated that rHeps clustered closely with cultured primary human hepatocytes (PHHs) (Fig. 1f).

rHeps lose the malignant characteristics of HCC cells The proliferation capability of rHeps was assessed by the CCK-8 assay and plate colony formation assay. We found that the proliferation of reprogrammed HCCLM3 and Huh-7 cells was dramatically reduced compared with that of control cells (Fig. 2a; Supplementary information, Fig. S5a). Moreover, reprogrammed HCCLM3 and Huh-7 cells exhibited extremely low migration ability in comparison with their counterparts (Fig. 2b). Many more senescent cells were detected in rHeps than in control cells, also reflecting loss of malignant features of rHeps (Fig. 2c). The effects of the three factors on liver cancer stem cells (LCSCs) was assessed by a spheroid formation assay, which showed few LCSC to be present in the rHeps population, suggesting that LCSCs had differentiated during induction (Supplementary information, Fig. S5b). Importantly, rHeps did not form colonies in soft agar after 3 weeks in culture, implying that rHeps might lose tumorigenicity (Fig. 2d). To test this hypothesis, the reprogrammed HCCLM3 or Huh-7 cells and their control counterparts were inoculated subcutaneously into both dorsal flanks of NOD/ SCID mice. At day 14 post inoculation, tumor onset was observed in the NOD/SCID mice inoculated with HCCLM3 control cells, and all mice bearing HCCLM3 control cells developed tumor nodules by day 40. Consistently, the Huh-7 control cell xenografts generated tumors as early as day 7 , and all mice implanted with Huh-7 control cells developed tumor nodules by day 30 . Importantly, none of the NOD/SCID mice inoculated with reprogrammed HCCLM3 or Huh-7 cells exhibited tumor nodules up to 60 days post inoculation (Fig. 2e, f) suggesting that the rHeps had lost the malignant phenotype.

rHeps recover specific functions of hepatocytes

Since rHeps exhibited certain morphological characteristics of hepatocytes (Fig. 3a), we wanted to know whether they accomplished unique hepatic functions. Real-time PCR assays showed that a set of hepatocyte-specific genes were efficiently induced in reprogrammed HCCLM3 and Huh-7 cells (Fig. 3b, c). Moreover, rHeps displayed numerous hallmark features of mature hepatocytes, including glycogen storage (Fig. 3d) and intake of acetylated low density lipoprotein (ac-LDL) (Fig. 3e). In addition, the urea levels in the supernatants of rHeps were much higher than those of control cells (Fig. 3f) indicating the capability of rHeps for urea production capability. These results suggest that rHeps re-aquire specific functions of mature hepatocytes.

rHeps exhibit induced CYP enzyme and drug metabolism activity Considering that detoxification is a unique characteristic of the mature hepatocyte, we evaluated the detoxification ability of rHeps. Our microarray data revealed that a set of drug metabolism-associated genes were notably induced in rHeps compared with control cells (Fig. 4a). Cytochrome P450 (CYP450) enzymes in hepatocyte are the predominant enzymes responsible for hepatic detoxification, and their expression levels and drug response have been irreplaceably utilized to assess the drug metabolism ability of hepatocytes. ${ }^{15}$ Our results showed that the key CYP450 enzymes, including CYP1A2, CYP2C19 and CYP3A4, were remarkably up-regulated in rHeps (Fig. 4b, c). Transcription of CYP450 enzymes and many other drug metabolism enzymes are usually controlled by nuclear receptors, including PXR, GR, and RXRB. The expression levels of these nuclear receptors were much higher in rHeps than that in control cells (Supplementary information, Fig. S6a, b). Membrane transporter-mediated biliary excretion is another unique function of the mature hepatocyte, which is indispensable for clearance of xenobiotics. ${ }^{16}$ The expression levels of the key transporter genes were significantly induced in rHeps compared with the control cells (Fig. 4d, e; Supplementary information, Fig. S6c, d). When the drug response of rHeps to CYP inducers was evaluated, we found that omeprazole and rifampicin treatment markedly induced the mRNAs of CYP1A2, CYP2C19, CYP2C8 and CYP3A4 (Fig. 4f, g). Furthermore, in the assay for CYP activities, rHeps showed the CYP enzyme-dependent metabolism of phenacetin and testosterone (Fig. 4h, i). Taken together, these results suggest that rHeps should possess the detoxification ability of mature hepatocyte.

HNF1A, HNF4A and FOXA3 induce HCC cell reprogramming in vivo

To explore whether the combination of HNF1A, HNF4A and FOXA3 could induce the reprogramming of $\mathrm{HCC}$ cells in vivo, we established cell-derived xenograft (CDX) and patient-derived xenograft (PDX) models. We found that CDXs treated with Ad-3F were significantly smaller than those treated with Ad-GFP at successive time intervals, consistent with final weight measurement (Supplementary information, Fig. S7a). Consistent results were achieved using PDX models from different HCC patients (Fig. 5a, b; Supplementary information, Fig. S7b). Moreover, Ad-3Ftreated mice exhibited significantly longer survival than control mice (Fig. 5c). Real-time PCR and western blot assays demonstrated the induction of human ALB and reduction of AFP in PDXs treated with Ad-3F compared to the PDXs treated with Ad-GFP (Fig. 5d). As anticipated, many hepatocyte-specific genes were efficiently induced in Ad-3F-treated PDXs compared with control PDXs (Fig. 5e). More importantly, rHeps, which exhibited glycogen storage and accumulation of neutral triglycerides and lipids $(\sim$ $10 \%$ area in total) were scattered throughout the population of Ad-3F-treated PDXs, suggesting the conversion of HCC cells to 
a

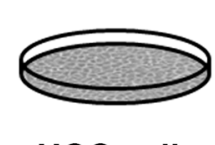

HCC cells
HNF1A , HNF4A , FOXA3
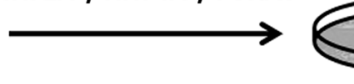

rHeps
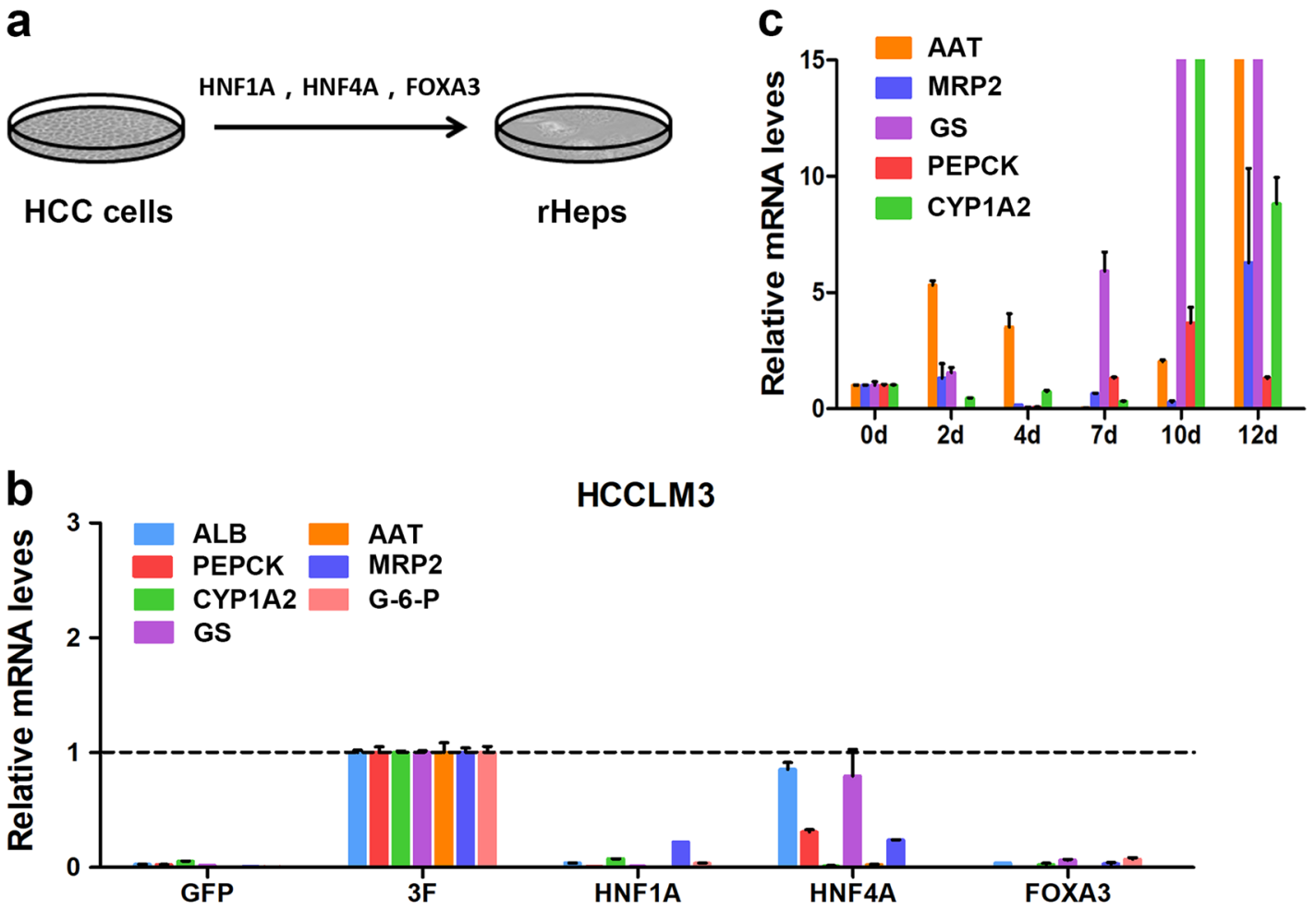

d
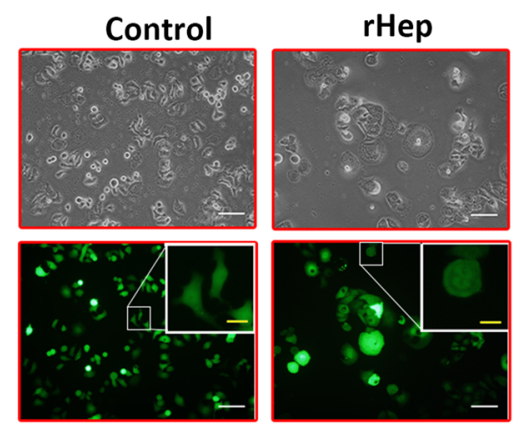

e

Albumin secretion
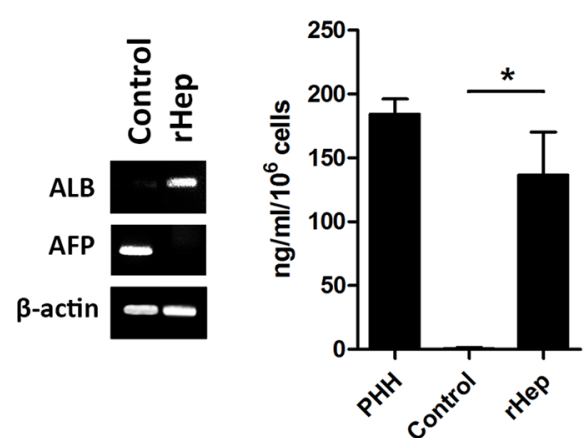

f

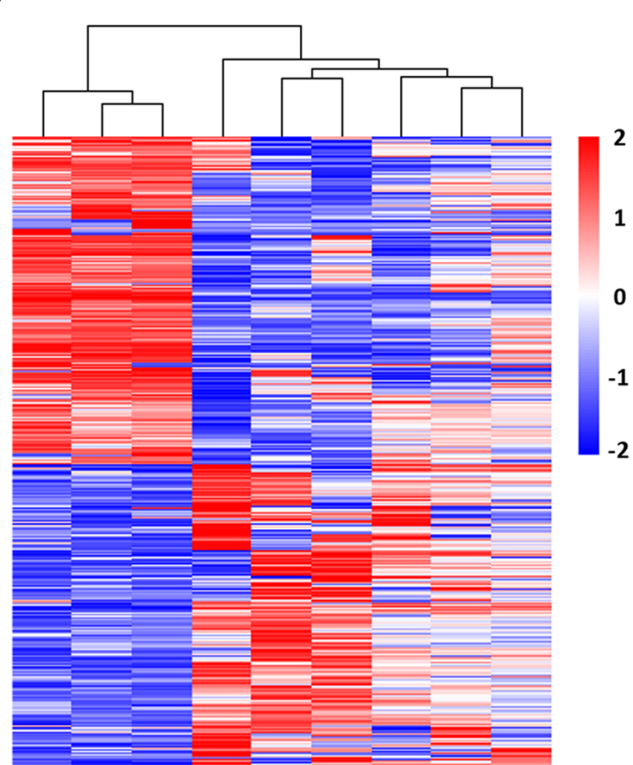

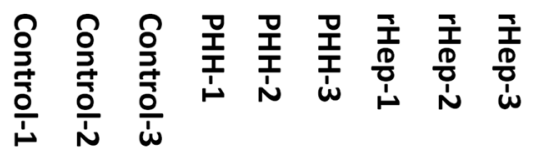

Fig. 1 HNF1A, HNF4A and FOXA3 induce HCC cell reprogramming. a The experimental design for the induction of rHeps. HCC cells were infected with adenovirus expressing HNF1A, HNF4A and FOXA3 (each at MOI = 10). rHeps were characterized 10-14 days after induction. b Hepatic gene expression in HCCLM3 cells induced by different adenoviruses expressing HNF1A, HNF4A or FOXA3 ( $n=3$ ). Data are normalized to 3F (HNF1A, HNF4A and FOXA3). c Hepatic gene expression gradually increased during conversion of HCCLM3 cells to rHeps. Expression levels of the indicated genes were analyzed by real-time PCR $(n=3)$. Data are normalized to day 0 . $\mathbf{d}$ Hepatocyte-like morphology of rHeps derived from HCCLM3 cells observed by fluorescence microscopy. Scale bar in white, $100 \mu \mathrm{m}$. e Expression of ALB and AFP in rHeps derived from HCCLM3 cells was analyzed by RT-PCR (left), and ALB secretion was measured by ELISA (right) ( $n=3$ ). Primary human hepatocytes (PHHs) cultured for 2 days were used as positive control. $\mathbf{f}$ Global gene expression profile analysis of rHeps derived from HCCLM3 cells and Ad-GFP-treated control cells by CDNA microarray. PHHs were used as the positive control. Hierarchical clustering showed that rHeps grouped together with PHHs. * indicates $P<0.05$ 
a

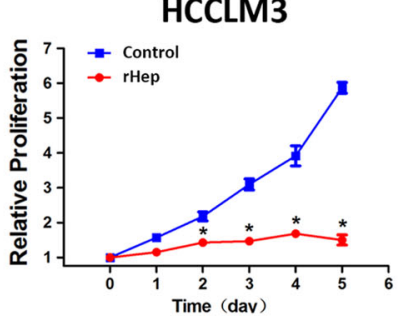

Huh-7

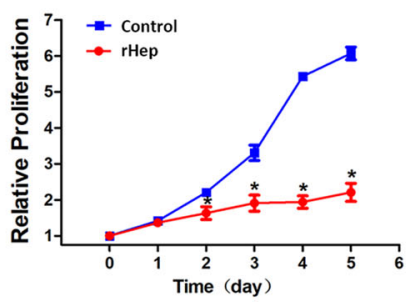

C
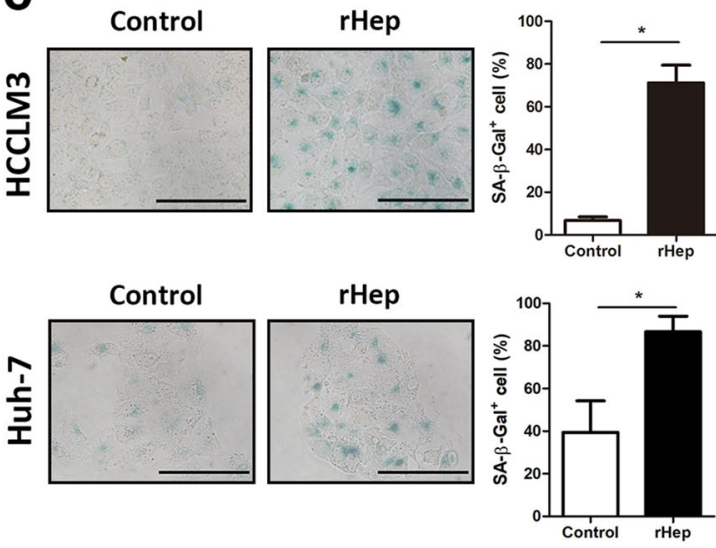

e

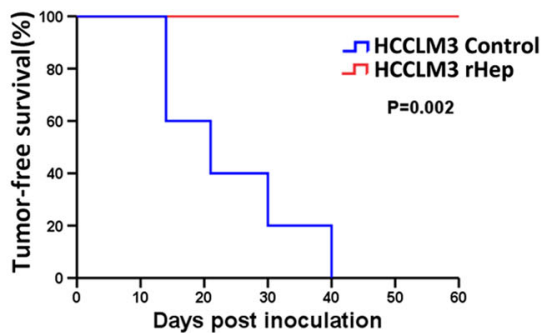

f

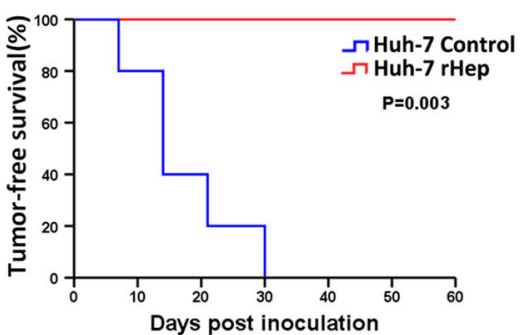

b
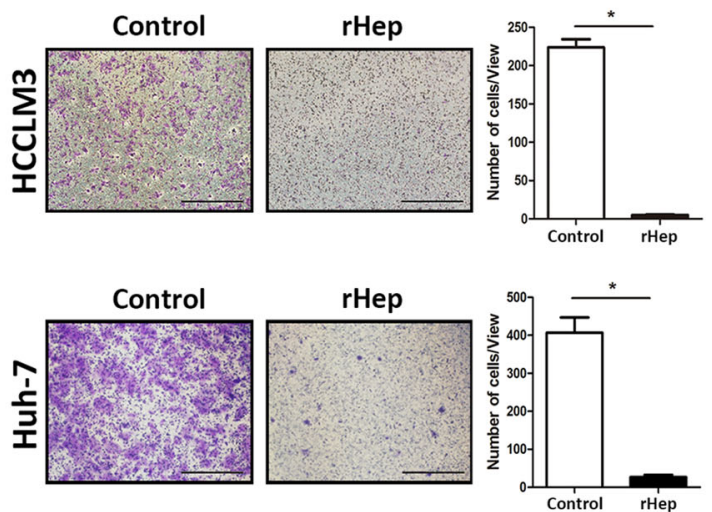

d
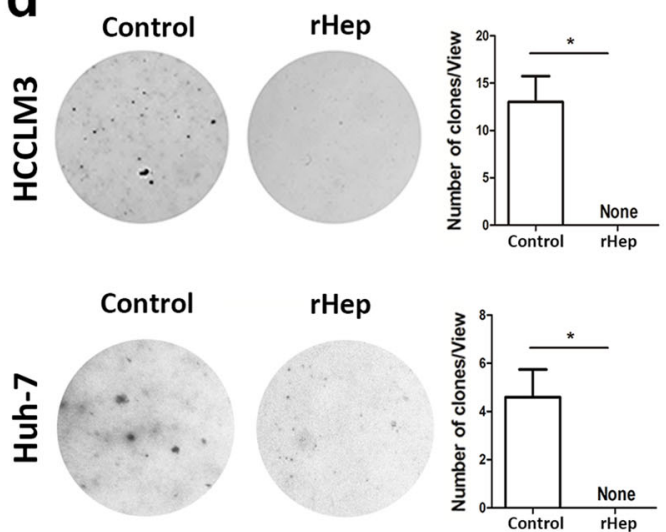

Fig. 2 rHeps lose the malignant phenotypes. a The proliferation of rHeps and control cells was compared by the CCK8 assay; $n=3$ for each group. b Cell migration assay was performed in Transwell filter chambers $(n=3)$ and representative images are shown (left). The number of migrated cells was calculated (right). Scale bar, $100 \mu \mathrm{m}$. c Senescent cells were detected by $\beta$-gal staining $(n=3)$ and representative images are shown (left). The percentage of senescent cells was calculated (right). Scale bar, $100 \mu \mathrm{m}$. d Cells were seeded in soft agar for three weeks to evaluate the colony formation ability of $\mathrm{rHeps}(n=3)$. Representative images are shown (left). Colony number is indicated to the right. Scale bar, $100 \mu \mathrm{m}$. e, f The difference in tumor free survival between control cells- and rHep-inoculated mice was compared by Kaplan-Meier analysis (left, $n=5$, log-rank test). Subcutaneous implantation of rHeps and control cells is shown to the right. ${ }^{*}$ indicates $P<0.05$ 


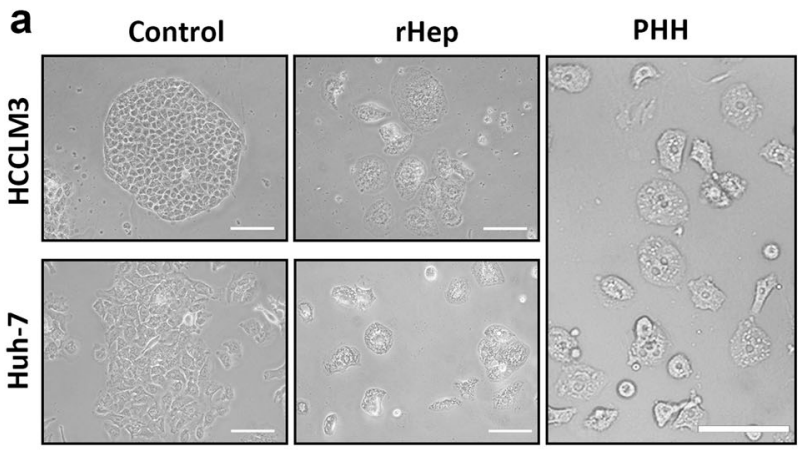

\section{b}
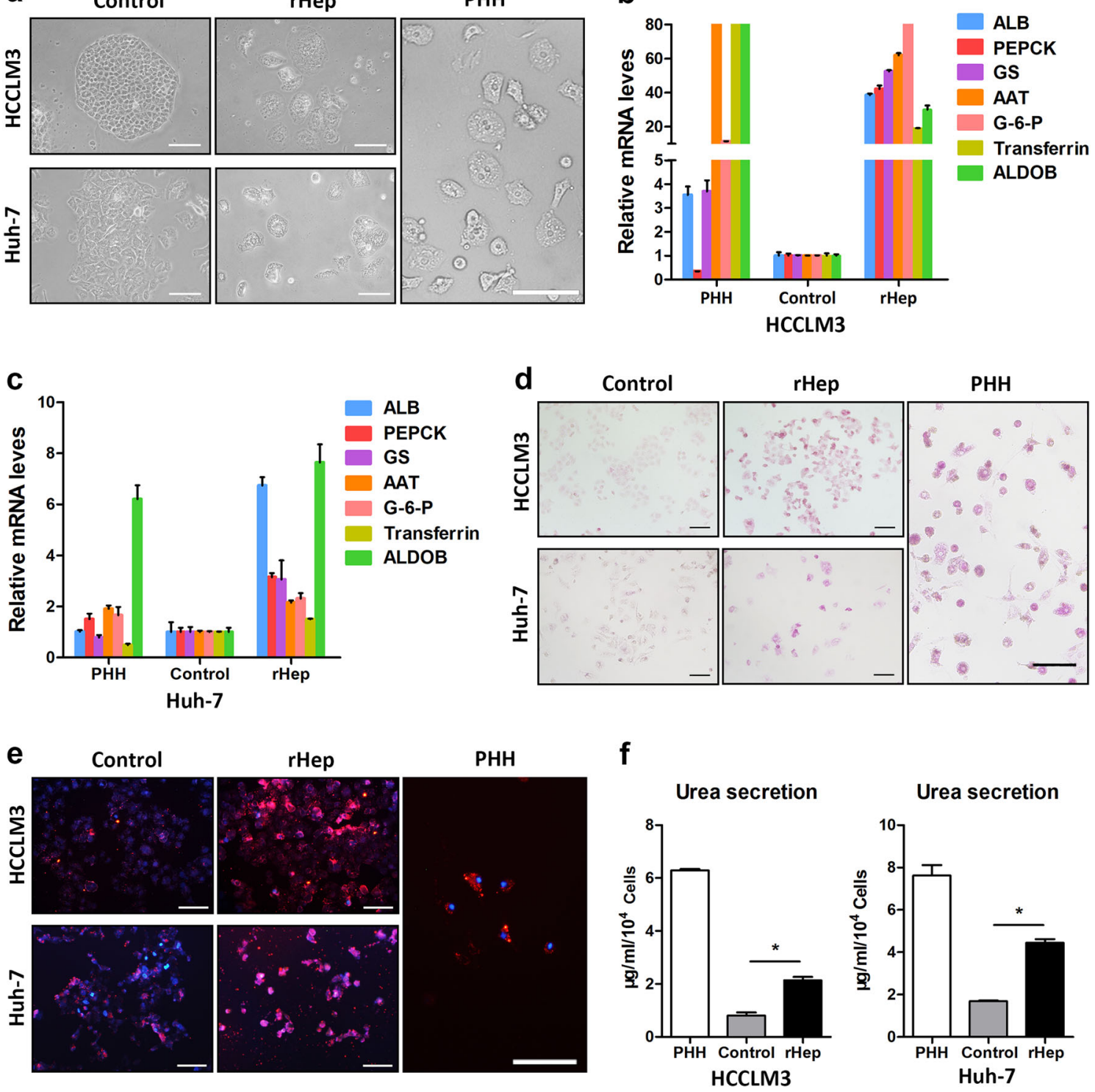

Fig. 3 rHeps recover the unique functions of hepatocytes. a The morphological comparison between rHeps and control cells. Scale bar, $100 \mu \mathrm{m}$. Primary human hepatocytes were used as positive control. b The levels of hepatic genes in HCCLM3-derived rHeps were determined by real-time PCR. PHHs cultured for 2 days were used as normal control. Data are normalized to HCCLM3 control cells $(n=3)$. c The hepatic genes expressed in Huh-7-derived rHeps were determined by real-time PCR. PHHs cultured for 2 days were used as normal control. Data are normalized to Huh-7 control cells $(n=3)$. d Glycogen storage in rHeps was confirmed by Periodic-Acid Schiff staining. Scale bar, $50 \mu$ m. Primary human hepatocytes were used as positive control. e LDL uptake ability of rHeps was analyzed using the Dil-ac-LDL fluorescent substrate (red). Scale bar, $100 \mu \mathrm{m}$. Primary human hepatocytes were used as positive control. f Urea production by rHeps was measured using the QuantiChrom ${ }^{\text {TM }}$ Urea Assay Kit $(n=3)$. PHHs cultured for 2 days were used as normal control. ${ }^{*}$ indicates $P<0.05$

rHeps in vivo (Fig. 5f). Considering the particular advantage of adeno-associated virus (AAV) in gene therapy, we also tested the therapeutic effects of AAV-3F using the PDX models, and consistent results were achieved (Supplementary information, Fig. S8).

rHeps could reconstruct the hepatic plate and liver lobules Transplanted hepatocytes can repopulate the destroyed area of liver in fumarylacetoacetate hydrolase-deficient $\left(\mathrm{Fah}^{-1-}\right)$ mice. This has been regarded as a golden criterion for functional identification of hepatocytes. To further validate the unique function of rHeps in vivo, we transplanted rHeps into the livers of $\mathrm{Fah}^{-1-} \mathrm{Rag2}^{-1-}$ mice through spleen injection. Strikingly, all mice transplanted with control cells exhibited xenografted HCCs whereas no tumors were detected in rHep recipient mice (Fig. 6a). rHeps could be recognised in the livers of rHep recipient mice through their larger size and less eosinophilic appearance (Fig. 6b). Moreover, H\&E staining of the chimeric liver showed no loss of normal tissue organization in rHep engrafted livers, suggesting that rHeps could be successfully engrafted and propagated in $\mathrm{Fah}^{-1-} \mathrm{Rag}^{-1-}$ mice, thus contributing to hepatic plate formation and maintenance of liver lobule integrity (Fig. 6b). To further confirm that the repopulating rHeps exert hepatic function, we examined the expression of human Fah, ALB and AAT by staining serial sections from chimeric mice livers. As expected, this showed that the proliferated rHeps were positive for human Fah, ALB and 
a
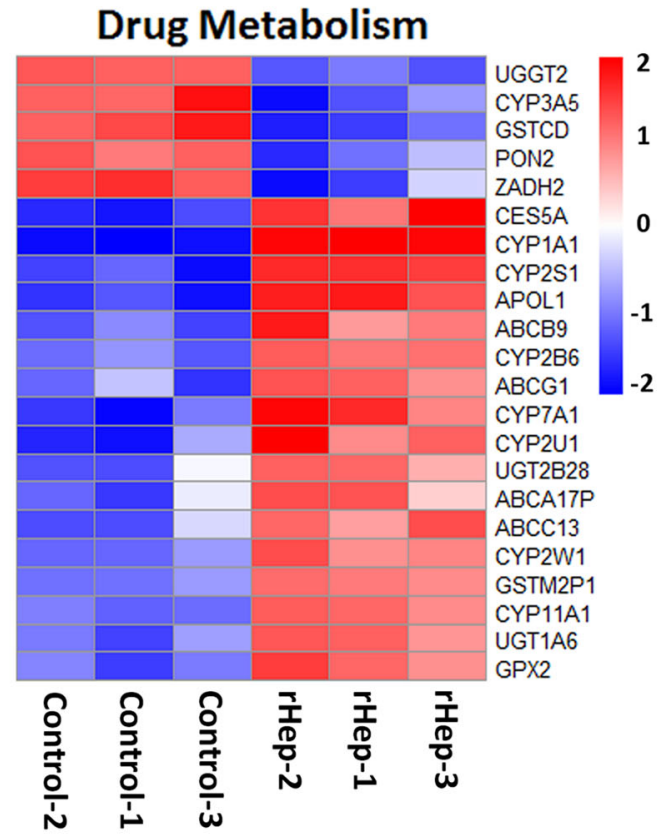

d

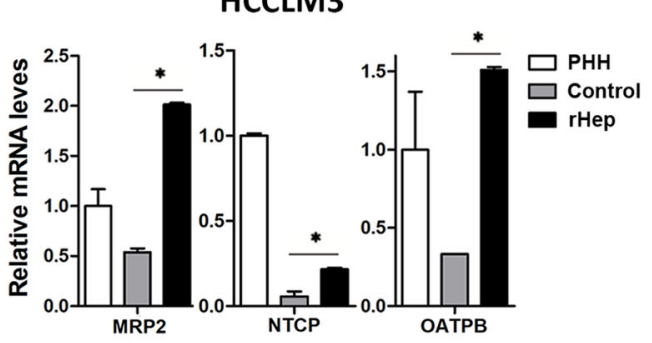

f

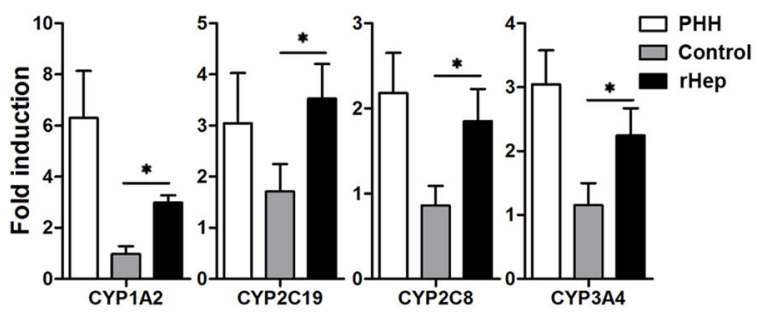

h

Phenacetin
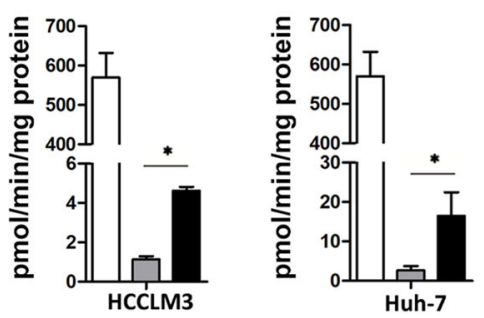

AAT (Fig. 6b). Consistently, we detected human ALB in the sera of rHep recipient mice but not in control mice, further validating the normal function of rHeps in vivo (Fig. 6c). We then evaluated the efficiency of liver repopulation by rHeps by examining human Fah expression in the livers of $\mathrm{Fah}^{-1-} \mathrm{Rag2}^{-1-}$ mice. This revealed that b
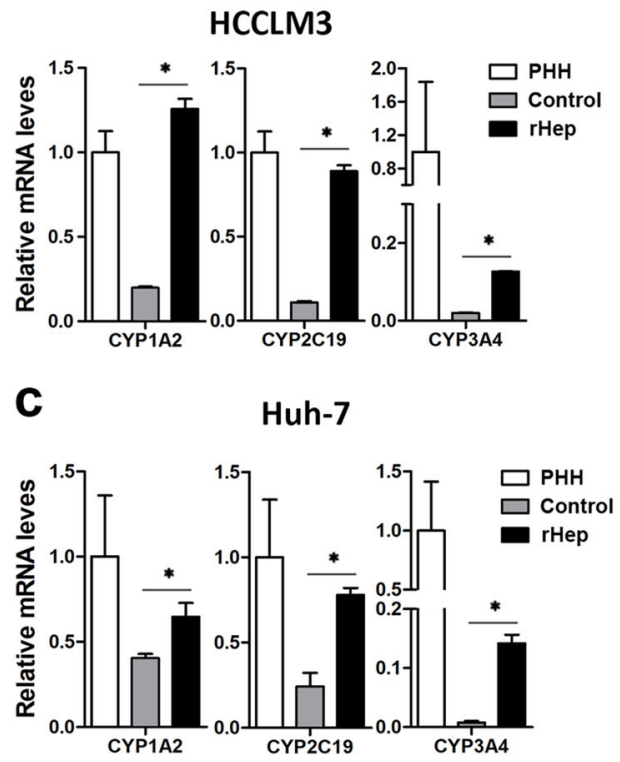

e

Huh-7

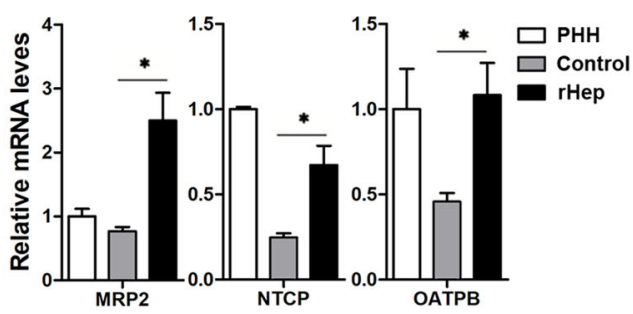

g

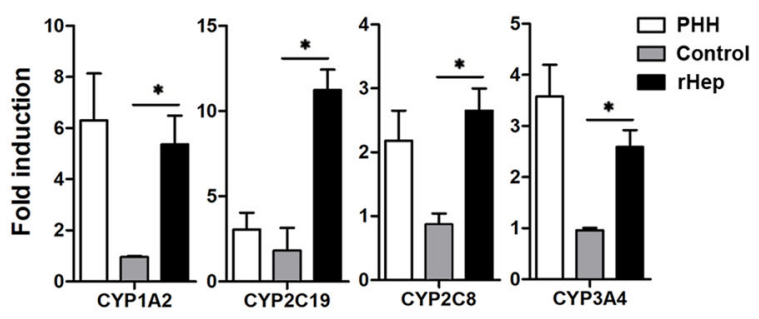

i

Testosterone

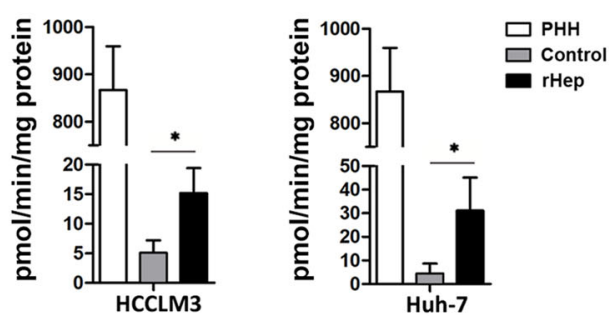

Fah-positive rHeps contributed as much as $4.69 \%$ to the chimeric livers in $\mathrm{Fah}^{-1-} \mathrm{Rag}^{-1-}$ mice (Fig. 6d, e; Supplementary information, Fig. S9). Our previous data showed that transplantation with $\mathrm{PHH}$ extended the life of recipient $\mathrm{Fah}^{-1-} \mathrm{Rag}^{-1-}$ mice. ${ }^{9}$ Here we found that $\mathrm{Fah}^{-1-} \mathrm{Rag2}^{-1-}$ mice transplanted with control cells 
Fig. 4 rHeps possess CYP enzyme induction and drug metabolism activity. a Drug metabolism-associated genes differentially expressed in rHeps compared to control cells were selected from cDNA microarray assay. b, c Expression of CYP genes in rHeps and the control cells was compared by real-time PCR $(n=3)$. Data are normalized to the PHH control cells. $\mathbf{d}$, e Expression of drug transporter genes in rHeps and the control cells was compared by real-time PCR $(n=3)$. Data are normalized to PHH controls. $\mathbf{f}, \mathbf{g}$ The mRNA levels of the induced CYP enzymes were measured by real-time PCR $(n=3)$. CYP1A2 and CYP2C19 were induced by omeprazole (10 $\mu \mathrm{M})$. CYP2C 8 and CYP3A4 were induced by rifampicin $(10 \mu \mathrm{M})$. The fold induction in control cells, rHeps and PHHs was normalized to the levels in cells without inducer treatment. $\mathbf{h}$, $\mathbf{i}$ The metabolic products of phenacetin (phenacetin o-deethylation, assay for CYP1A2 activities) and testosterone (testosterone $6 \beta$-hydroxylation, assay for CYP3A4 activities) were determined by liquid chromatography-tandem mass spectrometry. Primary human hepatocytes were used for the positive control. * indicates $P<0.05$

were dead within 55 days of NTBC removal. In contrast, transplantation of rHep cells significantly improved the survival of $\mathrm{Fah}^{-1-} \mathrm{Rag2}^{-1-}$ mice (Fig. 6f).

Molecular mechanisms underlying conversion of HCC cells to rHeps

To explore the expression pattern of HNF1A, HNF4A and FOXA3 during HCC cell reprogramming, we measured the transcripts of these factors at different time points by RT-PCR. As shown in Fig. 7a, b, expression of these three factors peaked at Day 2 or Day 3 and then gradually decreased. Strikingly, the levels of these factors began to elevate at Day 6 or earlier and displayed a second boost (Fig. 7a, b). HNF1A, HNF4A and FOXA3 belong to the hepatocyte nuclear factor family, which consists of 6 transcription factor subfamilies indispensable for hepatocyte differentiation and hepatic function maintenance. Our bioinformatics analysis identified a set of HNFs involved in the regulatory network centered by HNF1A, HNF4A and FOXA3 (Fig. 7c). These HNFs could constitute an interactive network that synergistically regulates the transcription of a large number of hepato-specific genes. As anticipated, our gene array data revealed that a number of hepatocyte nuclear factors were up-regulated in rHeps compared with control cells (Fig. 7d, e). Consistently, real-time PCR assay of PDXs demonstrated that most of those HNFs were up-regulated by the combination of HNF1A, HNF4A and FOXA3 in vivo (Fig. 7f). Collectively, these data suggest that exogenous HNF1A, HNF4A and FOXA3 in HCC cells might initiate the endogenous expression of various hepatocyte nuclear factors, which promote the conversion of HCC cells to hepatocyte-like cells. Materials and methods are available in Supplementary information, Data S1.

\section{DISCUSSION}

Hepatocellular carcinoma is the sixth most common cancer and the third leading cause of cancer death globally. ${ }^{17}$ Despite advances in the diagnosis and surgical treatment of the disease during the past several decades, the prognosis for patients remains disappointing due to the extreme resistance of HCC cells to chemotherapeutics or radiotherapy. ${ }^{18,19}$ Therefore, novel strategies for HCC treatment are urgently required. Here we report for the first time that simultaneous expression of HNF1A, HNF4A and FOXA3 can convert liver cancer cells into hepatocytelike cells. Mechanistically, we found that exogenous expression of HNF1A, HNF4A and FOXA3 in HCC cells synergistically initiated the endogenous expression of various hepatocyte nuclear factors, which facilitated the conversion of HCC cells to hepatocyte-like cells.

Hepatocyte nuclear factor is a type of liver-enriched transcription factor that plays important roles in hepatocyte differentiation and the maintenance of hepatic function. ${ }^{20}$ We previously observed that expression of HNF4A and HNF1A was decreased during HCC development. ${ }^{21-23}$ HNF4A delivery hampered DEN (diethylnitrosamine)-induced rat hepatocarcinogenesis and reduced HCC metastasis. ${ }^{21,23}$ In addition, our previous study demonstrated that forced HNF1A expression repressed the proliferation of HCC cells. ${ }^{22}$ However, the expression of HNF1A or HNF4A was not reduced in all HCC cases. Indeed, we found that the respective levels of HNF1A and HNF4A were not decreased in 25 and $37 \%$ of patient HCCs reflecting the extreme heterogeneity of HCCs (Supplementary information, Fig. S10). Nevertheless, 95\% of patient HCCs exhibited the reduction of HNF1A, HNF4A or FOXA3 alone or their pairwise combinations, and all three factors were reduced in $50 \%$ of the patient HCCs, suggesting an advantage of the combinational use of the three factors. Most importantly, we have observed, for the first time, that combination of the three factors achieved a synergistic effect in the conversion of HCC cells to rHeps, which lost malignant phenotypes.

Although there have been intensive investigations of the reprogramming of somatic cells into iPSCs or other cell lineages, few studies have focused on the reprogramming of cancer cells. In previous studies, distinct types of cancer cells, including melanoma cells, lung cancer cells and gastrointestinal cancer cells, have been reprogrammed into stem cell-like pluripotent cells by the nuclear transfer technique or by delivery of OSKM factors (Oct4, Sox2, KIf4 and c-Myc). ${ }^{24-26}$ Our present study has shown that forced expression of HNF1A, HNF4A and FOXA3 not only induces cell cycle arrest and apoptosis (data not shown) but also converted HCC cells into rHeps, which lost malignant characteristics. In addition, we analyzed the cancer-related genes in our microarray data, and found some oncogenic genes were suppressed and a few tumor suppressor genes were upregulated in rHeps compared with control cells, findings that warrant further investigation (Supplementary information, Table S1). More importantly, rHeps acquired typical hepatic phenotypes, including epithelial morphology; global expression patterns of hepatocyte genes and specific biomarkers; and unique hepatic functions. To some extent, this process could be regarded as another type of cell reprogramming, in the disordered state of cancer cells was reprogrammed into the ordered state of hepatocytes. Nevertheless, karyotype analysis revealed that both the rHeps and the original HCC cells were both highly aneuploid. In fact, over half of normal mature hepatocytes in mice and humans are also highly aneuploid. ${ }^{27,28}$ Therefore, whether normalization of the karyotype is important in to achieve full reprogramming of HCC cells into rHeps remains unclear and is worthy of further exploration.

$\mathrm{Fah}^{-1-}$ mice are defective in tyrosine metabolism and require a supply of 2-(2-nitro- 4-trifluoro-methylbenzyol)-1, 3cyclohexanedione (NTBC) for survival. ${ }^{29,30}$ After NTBC withdrawal, $\mathrm{Fah}^{-1-}$ mice suffer from progressive liver failure until the death. Intrasplenic transplantation of wild-type hepatocytes can repopulate the livers of $\mathrm{Fah}^{-1-}$ mice. This has been regarded as a golden criterion to identify functional hepatocytes. ${ }^{29,30}$ The use of immunodeficient $\mathrm{Fah}^{-1-} \mathrm{Rag2}^{-/-}$mice for hepatocyte transplantation largely eliminates the possibility of immunological rejection in vivo. ${ }^{12,31}$ In the present study, we transplanted rHeps into $\mathrm{Fah}^{-1-} \mathrm{Rag}^{-1-}$ mice by spleen injection to validate the hepatic function of rHeps. Strikingly, we detected propagated rHeps imbedding in the livers of all rHep recipient mice to reconstitute hepatic lobules and exert hepatic functions. In contrast, transplantation of control cells gave rise to liver cancer in all $\mathrm{Fah}^{-1-} \mathrm{Rag2}^{-1-}$ mice. To our knowledge, this is the first 

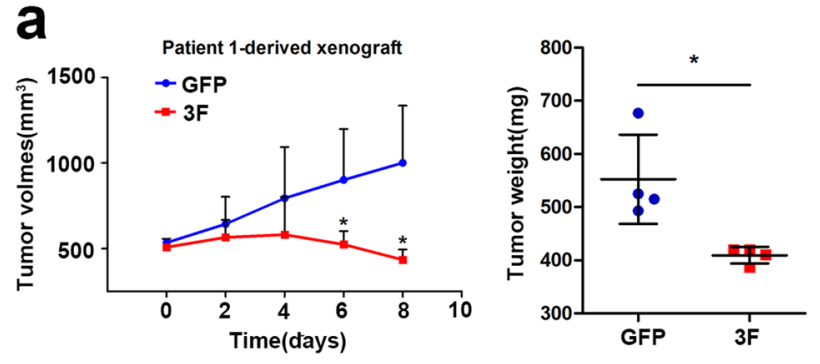

C
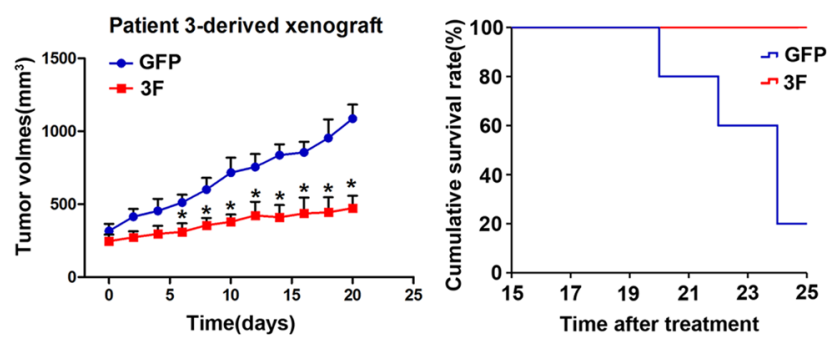

e

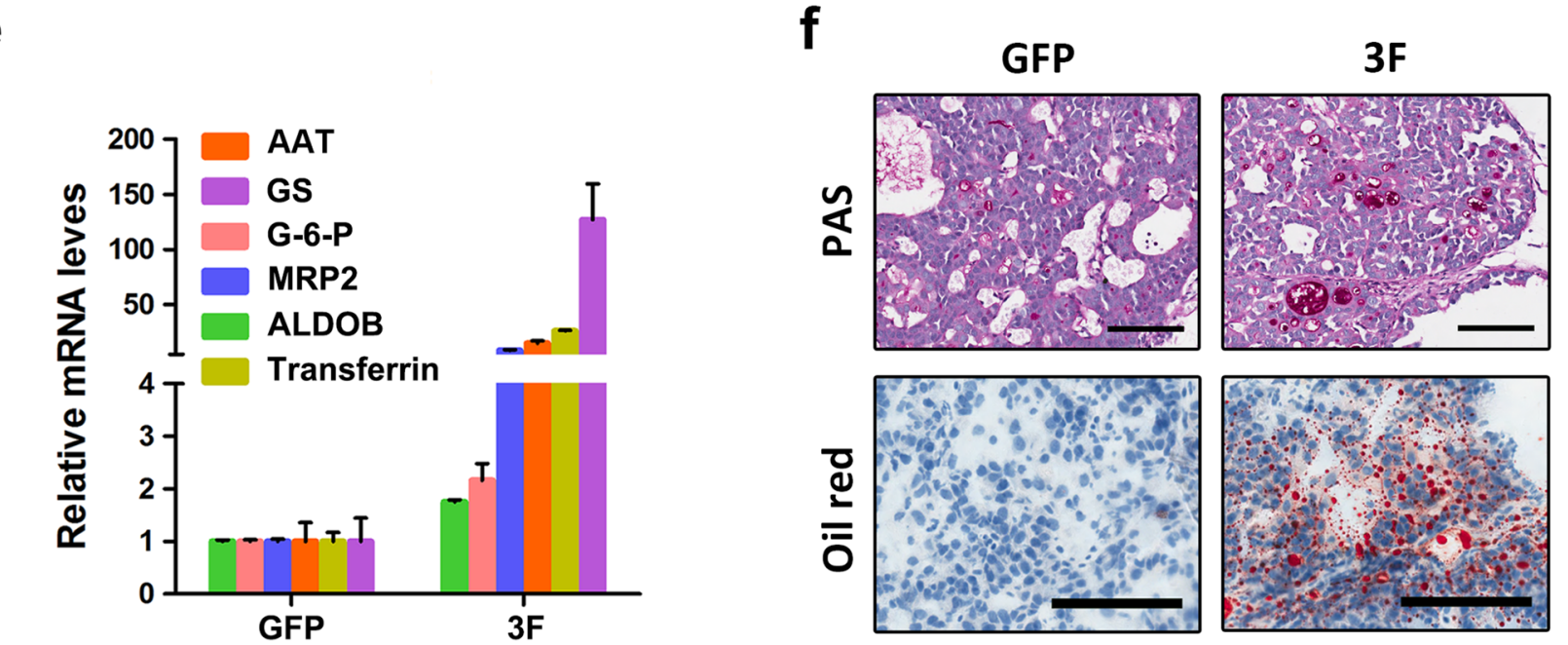

b
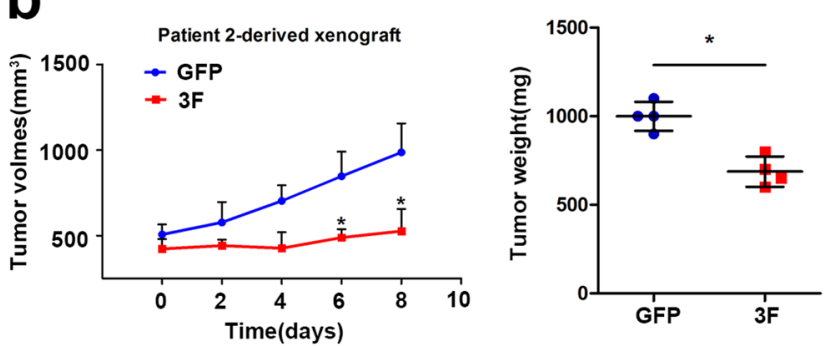

d

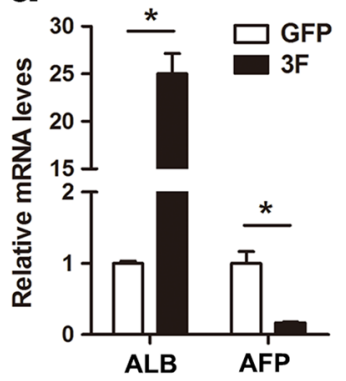

f

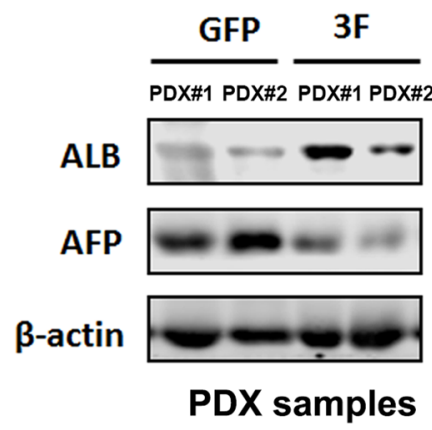

PDX samples

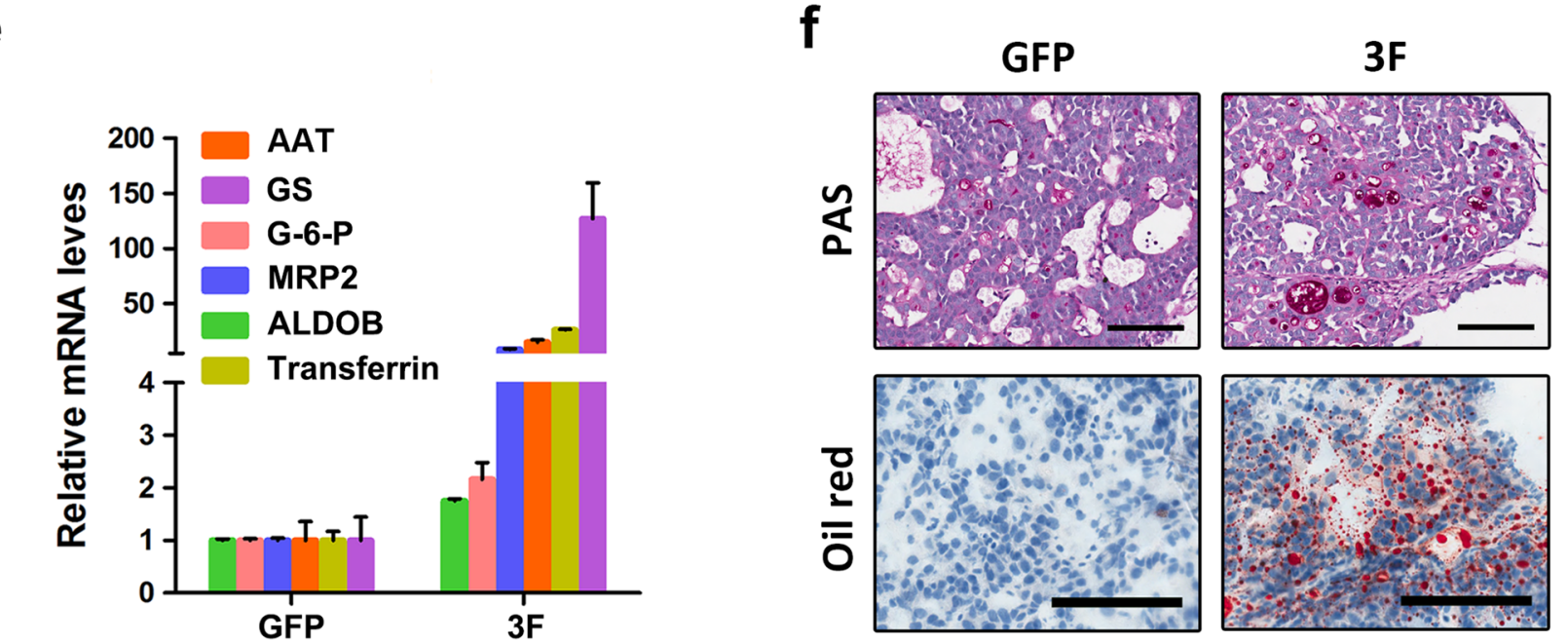

Fig. 5 Induction of rHeps by HNF1A, HNF4A and FOXA3 in vivo. a, b The size of patient 1- and patient 2-derived HCC xenografts was measured every other day and the difference in tumor volume between the Ad-3F-treated group and the Ad-GFP-treated group was observed (left). Tumor weight was measured after the sacrifice of nude mice (right). $\mathrm{n}=4$ for each group. c The size of patient 3-derived HCC xenografts was measured every other day and the difference in tumor volume between the Ad-3F-treated and the Ad-GFP-treated groups was observed (left). The difference in survival of mice between the Ad-3F-treated and the Ad-GFP-treated groups was compared using Kaplan-Meier analysis (right). $n=5$ for each group. d The expression of ALB and AFP in PDX samples was analyzed by real-time PCR and western blot. e Hepatic gene expression induced by Ad-3F in PDX was measured by real-time PCR in triplicate for one sample. f Glycogen accumulation was determined by PAS staining. Lipid accumulation was assessed by oil red $\mathrm{O}$ staining. Scale bar, $100 \mu \mathrm{m}$. * indicates $P<0.05$

report concerning the reprogramming of somatic cells from tumor cells to establish normal tissue architecture and behavior in vivo.

Great effort has been put into cell reprogramming and yet, the detailed molecular mechanisms underlying cell fate conversion remain largely unknown. In 2015, Kourtidis and colleagues reported that the pleckstrin homology domain containing A7 (PLEKHA7) could regulate several miRNAs (e.g. miR-30b) to suppress the expression of cell transforming markers such as SNAIL, MYC, and CCND1. Restoring the expression of PLEKHA7 or its downstream microRNAs in tumor cells might return tumorous cells to a benign state. However, we found no difference in PLEKHA7 expression between rHeps and control cells in our microarray analysis. ${ }^{32}$ Intriguingly, we did find that the expression of HNF1A, HNF4A and FOXA3 gradually decreased after an initial expression peak and then became re-elevated in a second wave. Our data suggests that exogenous HNF1A, HNF4A and FOXA3 were silenced in rHeps whereas endogenous factors were progressively induced. This might be explained by the exogenous factors initiating the expression of endogenous factors during the cell fate conversion. Hepatocyte nuclear factor represents a large family consisting of six subgroups, including HNF1, HNF3, HNF4, HNF6, CCAAT/enhancer binding protein (C/EBP) and D-binding protein (DBP). These transcription factors constitute an interactive, systemic network that synergistically regulates the expression of numerous hepato-specific and -enriched genes. ${ }^{20,33}$ Bioinformatics analysis together with our experimental validation revealed that a 
a
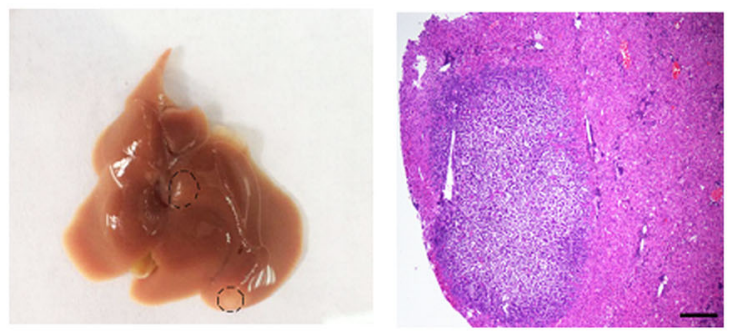

\begin{tabular}{lc}
\hline & $\begin{array}{c}\text { Tumor-bearing mice per } \\
\text { injected mice }\end{array}$ \\
\hline Control cells & $3 / 3$ \\
rHep cells & $0 / 6$ \\
\hline
\end{tabular}

b

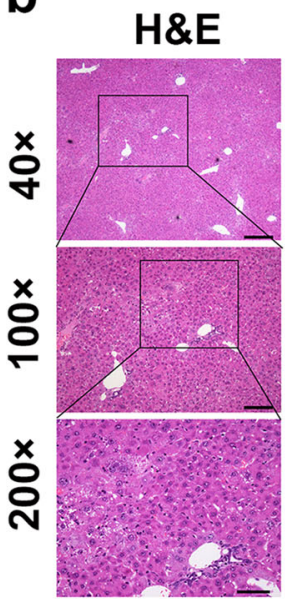

Fah

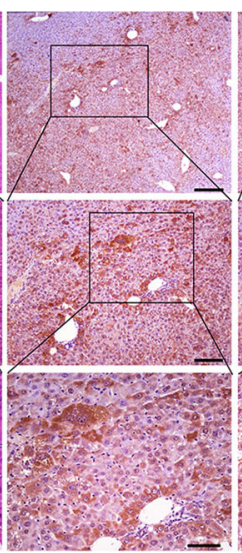

ALB
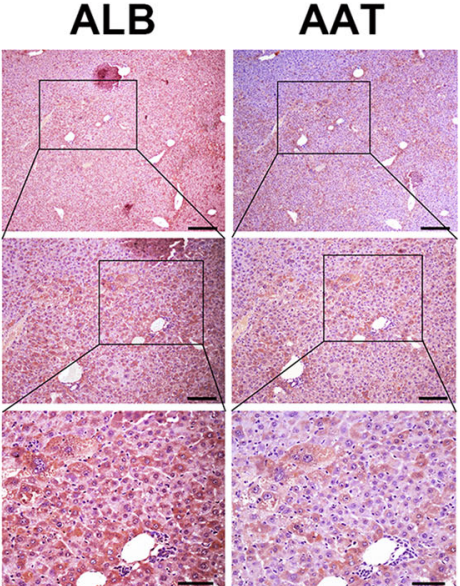

C

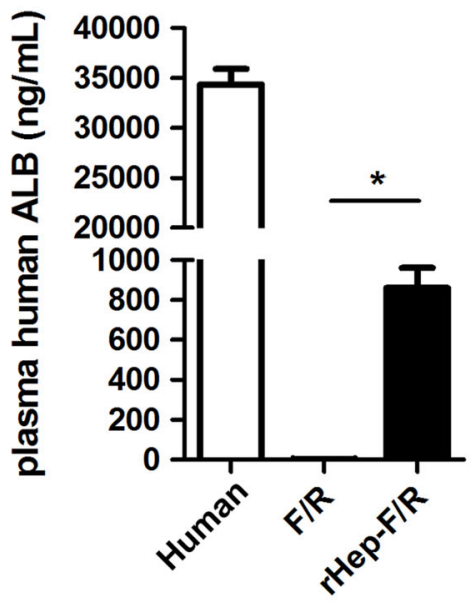

d
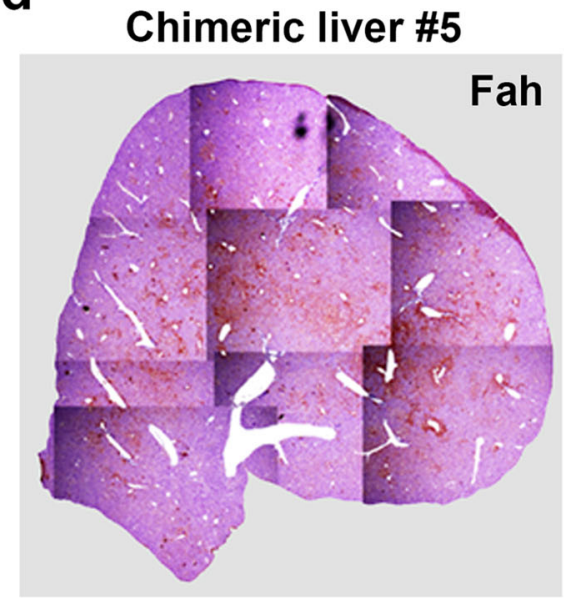

e

\begin{tabular}{ccc}
$\begin{array}{l}\text { No. of } \\
\text { recipient } \\
\text { mice }\end{array}$ & $\begin{array}{c}\text { Time after } \\
\text { transplantation } \\
\text { (weeks) }\end{array}$ & $\begin{array}{c}\text { Percentage of liver } \\
\text { repopulation by Fah } \\
\text { positive cells (\%) }\end{array}$ \\
\hline 1 & 4 & 0.03 \\
2 & 8 & 2.27 \\
3 & 14 & 2.99 \\
4 & 14 & 3.38 \\
5 & 14 & 4.55 \\
6 & 14 & 4.69 \\
\hline
\end{tabular}

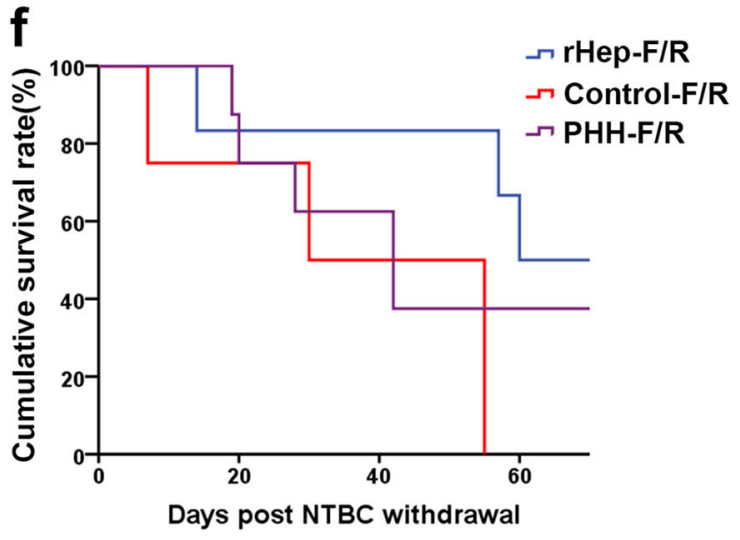

Fig. 6 Transplanted rHeps reconstructed the hepatic plate and liver lobules. a Respective HCCLM3-derived rHeps and control cells $\left(1.5 \times 10^{7}\right)$ were transplanted into the livers of $\mathrm{Fah}^{-1-} \mathrm{Rag}^{-1-}$ mice through spleen injection. Scale bars, $100 \mu \mathrm{m}$. b H\&E and immunohistochemical staining of human Fah, ALB and AAT in the livers of rHep recipient Fah ${ }^{-/-}$Rag $^{-1-}$ mice. c Human ALB levels in the sera of rHep-transplanted $\mathrm{Fah}^{-1-} \mathrm{Rag2}^{-1-}$ (F/R) mice were measured by ELISA. The sera from normal human and F/R mice were used as positive and negative controls, respectively. d Fah staining of the repopulated rHeps in $\mathrm{Fah}^{-1-} \mathrm{Rag}^{-1-}$ mice. Images of different section areas were merged into one using Adobe Photoshop CS5 (Adobe Systems). e The percentage of Fah-positive rHeps in the liver of each rHep recipient mouse was calculated and shown. f The Kaplan-Meier survival curve of $\mathrm{Fah}^{-1-} \mathrm{Rag}^{-/-}$mice that were transplanted with PHHs, control cells or rHep cells after NTBC removal. * indicates $P<0.05$

set of endogenous HNFs, including HNF1A, HNF4A and FOXA3, were significantly up-regulated in rHeps compared with the control HCC cells and that this may be responsible for the conversion of liver cancer cells to hepatocyte-like cells.
To conclude, our study reports the conversion of human liver cancer cells to hepatocyte-like cells via adenovirus-mediated delivery of HNF1A, HNF4A and FOXA3. This study not only extends our knowledge of cell reprogramming but also provides an 
a

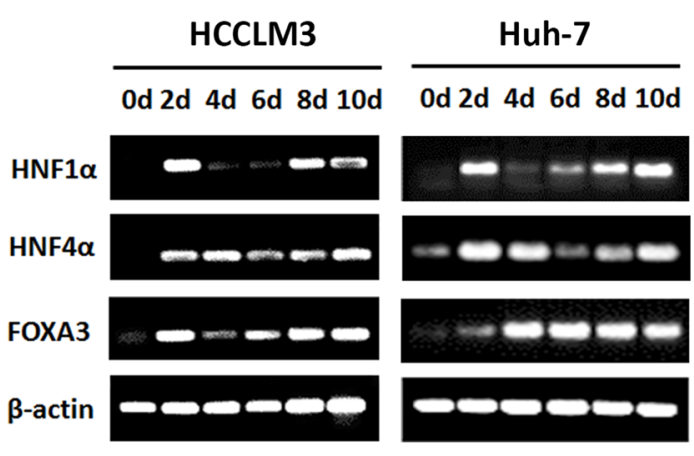

C

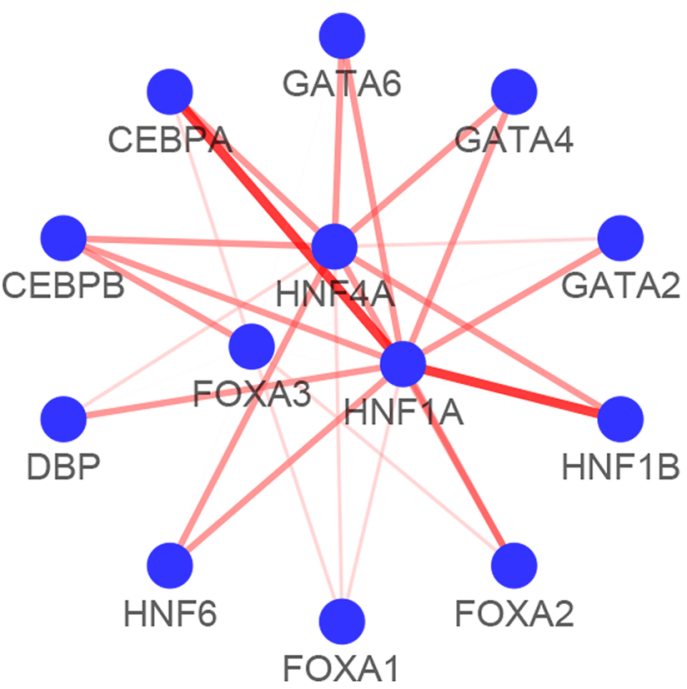

e

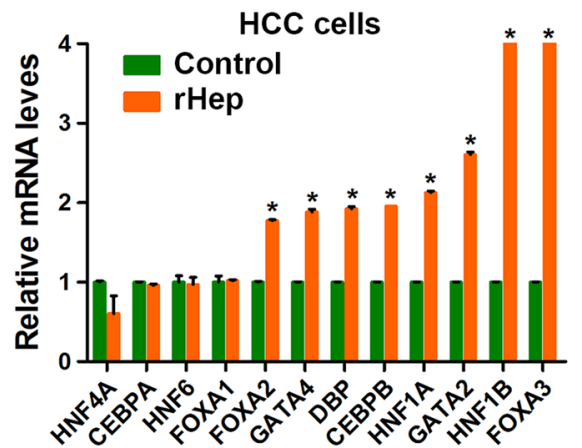

b
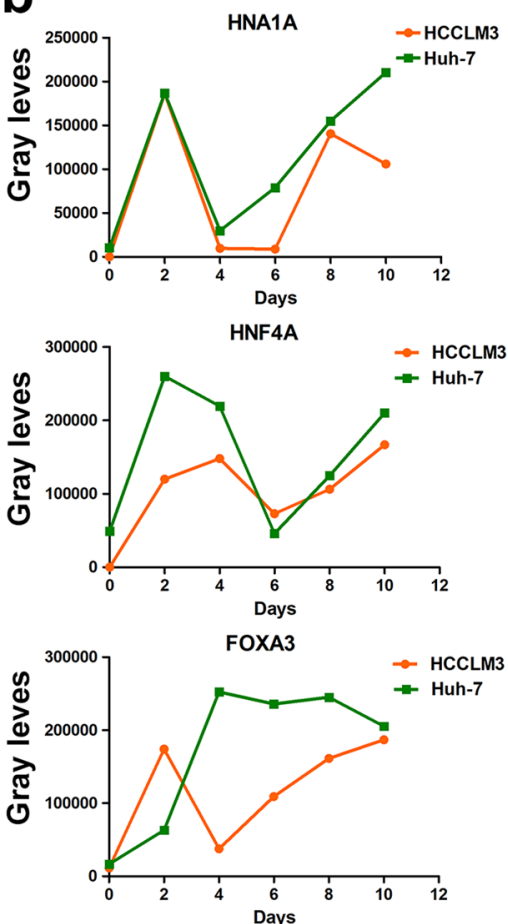

d

Hepatic Transcription Factors

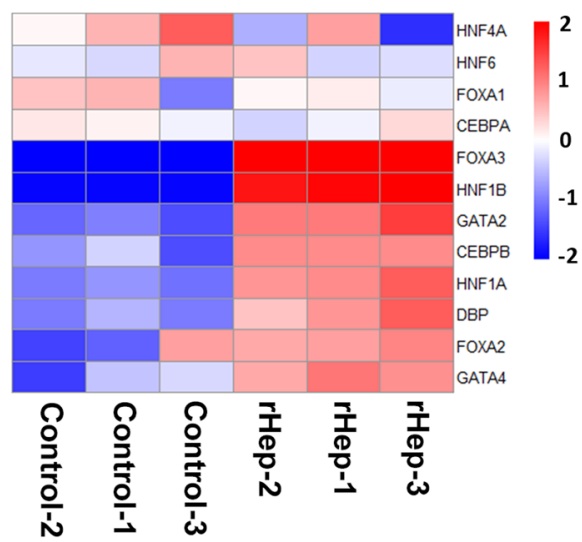

f

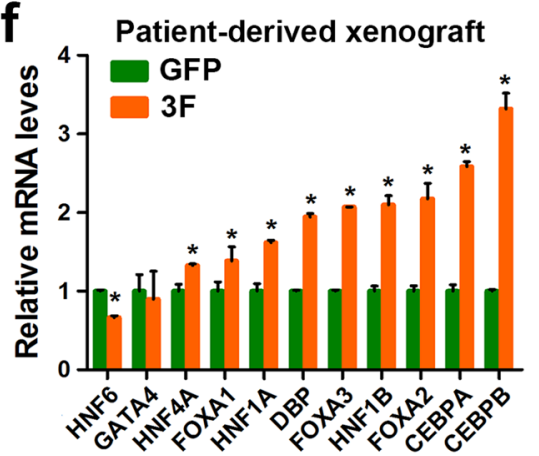

Fig. 7 Molecular mechanism of HCC cell conversion to rHeps. a The expression of HNF1A, HNF4A and FOXA3 during the induction of rHeps was analyzed by RT-PCR. $\mathbf{b}$ The expression pattern of HNF1A, HNF4A and FOXA3 based on the grey level quantification of electrophoretic bands in panel a. c The regulatory network of hepatocyte nuclear factors centered on HNF1A, HNF4A and FOXA3. The thickness and brightness of lines indicate the correlation degree between HNFs. d, e Hepatocyte nuclear factors that were differentially expressed in rHeps compared with control cells were extracted from CDNA microarray data. f Differential expression of HNFs in PDX samples treated by Ad-3F and Ad-GFP was achieved by real-time PCR in triplicate for one sample. ${ }^{*}$ indicates $P<0.05$ 
134

encouraging route towards HCC treatment. Whether this therapeutic strategy could be extended to the treatment of other solid tumors is worthy of investigation.

\section{MATERIALS AND METHODS}

\section{Adenovirus}

pCMV-FOXA3 and the control plasmid pCMV-GFP were cloned into the AdMax Shuttle vector according to the manufacturer's instructions (Microbix, Inc., Ontario, Canada). The Shuttle vector was co-transfected with the genomic plasmid into HEK-293 cells to achieve adenoviral packaging and the viral particles were further amplified and purified. AdHNF1A and AdHNF4A were prepared as previously described. ${ }^{21,22}$

Induction of rHeps and cell culture

Human HCC cell lines HCCLM3 and Huh-7 were from our laboratory stocks. The cells were infected with adenovirus carrying 3Fs (Ad-3F) or GFP (Ad-GFP) and cultured in DMEM with $10 \%$ fetal bovine serum (FBS) at $37^{\circ} \mathrm{C}$ in a humidified incubator containing $5 \% \mathrm{CO}_{2}$. The medium was changed every two days during the course of infection. rHeps were obtained and characterized 10-14 days after the viral infection. Their counterparts infected with Ad-GFP were considered to be control cells. Primary human hepatocytes were derived from normal tissue adjacent to hepatic hemangiomas.

\section{Microarray analysis}

Total RNAs were hybridized to whole-human gene expression microarray (Agilent) in accordance with the manufacturer's instruction. Data were normalized using Gene-Spring (Agilent).

Human albumin ELISA

Human albumin was measured by the Human Albumin ELISA Quantitation kit (Assay Pro) according to the manufacturer's instructions.

Hepatic function assays

The Periodic-Acid Schiff (PAS) staining kit was purchased from Sigma-Aldrich. Cells and paraffin sections of tissues were stained according to the manufacturer's instructions. For the LDL uptake assay, Dil-Ac-LDL (Invitrogen) was incubated with cells at concentration of $10 \mu \mathrm{g} / \mathrm{ml}$ for $4 \mathrm{~h}$ at $37^{\circ} \mathrm{C}$ before observation by fluorescence microscopy. Oil red O (Sigma-Aldrich) was carried out on frozen sections of tissue. Urea secretion was measured using the QuantiChrom ${ }^{\text {TM }}$ Urea Assay Kit (BioAssay System) and the discrepancy of cell growth was normalized by the CCK-8 assay kit.

CYP induction and metabolism assay

For the measurement of CYP enzyme induction, cells were cultured in DMEM for $24 \mathrm{~h}$ and then changed to fresh medium supplemented with omeprazole or rifampicin for $48 \mathrm{~h}$. Total RNA was isolated and CYP gene expression was measured by quantitative real-time PCR. To measure CYP metabolism activities, cells were treated with appropriate compounds for specific CYP metabolisms following omeprazole or rifampicin induction as indicated. The supernatants were then collected for the measurement of metabolized products by liquid chromatography-tandem mass spectrometry (Shimadzu LC 20, LEAP CTC HTS PAL and API 4000). ${ }^{9}$

Patient-derived xenograft (PDX) model

Fresh patient HCC tissue was cut into fragments and implanted subcutaneously into the flanks of nude mice. When the tumor growth reached a certain volume $\left(500 \mathrm{~mm}^{3}\right.$ or $\left.300 \mathrm{~mm}^{3}\right)$, the mice were subjected to intratumoral injection of Ad-3F or Ad-GFP $(1 \times$ $10^{9} \mathrm{pfu}$ in a $100 \mu \mathrm{l}$ volume) every other day. Tumor growth was monitored at the indicated time points. All HCC patients or their relatives signed informed consent forms, and the study was approved by the ethics committee of the Eastern Hepatobiliary Surgery Hospital.

Cell transplantation in $\mathrm{Fah}^{-1-} \mathrm{Rag}^{-1-}$ mice All animal experiments were performed in accordance with institutional regulations. rHeps were intrasplenically transplanted into $\mathrm{Fah}^{-1-} \mathrm{Rag2}^{-1-}$ mice after the withdrawal of NTBC water as previously described. ${ }^{12}$ FK506 was dissolved in the drinking water at $7.5 \mu \mathrm{g} / \mathrm{ml}$ and administered to adult mice to achieve a dose of $1 \mu \mathrm{g} / \mathrm{g}$ body weight per day. Surviving recipient mice were sacrificed to collect blood and liver samples. The blood of rHeptransplanted $\mathrm{Fah}^{-1-} \mathrm{Rag2}^{-1-}$ mice was collected for human serum ALB analysis. Mice were sacrificed by cervical dislocation and their livers harvested for IHC staining.

\section{Statistics}

The Student's T test was applied for calculating statistical probability as appropriate. For survival analysis, the Mantel-Cox log rank test was applied. Statistical calculations were performed using the Statistical Program for Social Sciences software (SPSS, IBM). For all statistics, data from at least three independent samples or repeated experiments were used.

\section{ACKNOWLEDGEMENTS}

The authors would like to thank Dr. Lijian Hui (Laboratory of Molecular Cell Biology, Shanghai Institutes for Biological Sciences, Chinese Academy for Sciences) for technical assistance and his suggestions in manuscript preparation. This work was supported by grants from the State Key Project of China (2017YFA0504503); the National Natural Science Foundation of China (81772582, 81572897 and 81471948); the Shanghai Pujiang Program (17PJD045); and Key Project of Shanghai Science and Technology Committee (17431906600).

\section{AUTHOR CONTRIBUTIONS}

J.D. and H.W. conceived the study. Z.C. and J.D. wrote the manuscript. Z.C., Z.H., Y.C. and C.Z. performed the majority of the experiments. Z.C., C.Z. and H.L. performed the in vitro experiments. Z.C. and X.L. performed the in vivo characterization. B.N., D.X. and T.Z. assisted with rHeps generation and characterization. Z.H., Y.C. and C.L. performed the transplantation into Fah-deficient mice and analyzed the results of the experiment. W.S., X.C. and W.X. provided technical support. Z.H. and Y.C. were involved in manuscript preparation.

\section{ADDITIONAL INFORMATION}

Supplementary information accompanies this paper at https://doi.org/10.1038/ s41422-018-0111-x.

Competing interests: The authors declare no competing interests.

\section{REFERENCES}

1. Gurdon, J. B. The developmental capacity of nuclei taken from intestinal epithelium cells of feeding tadpoles. J. Embryol. Exp. Morphol. 10, 622-640 (1962).

2. Blau, H. M., Chiu, C. P. \& Webster, C. Cytoplasmic activation of human nuclear genes in stable heterocaryons. Cell 32, 1171-1180 (1983).

3. Takahashi, K. \& Yamanaka, S. Induction of pluripotent stem cells from mouse embryonic and adult fibroblast cultures by defined factors. Cell 126, 663-676 (2006).

4. Feng, R. et al. PU.1 and C/EBPalpha/beta convert fibroblasts into macrophage-like cells. Proc. Natl Acad. Sci. USA 105, 6057-6062 (2008).

5. Huang, P. et al. Induction of functional hepatocyte-like cells from mouse fibroblasts by defined factors. Nature 475, 386-389 (2011).

6. Sekiya, S. \& Suzuki, A. Direct conversion of mouse fibroblasts to hepatocyte-like cells by defined factors. Nature 475, 390-393 (2011).

7. Vierbuchen, $T$. et al. Direct conversion of fibroblasts to functional neurons by defined factors. Nature 463, 1035-1041 (2010).

8. Pang, Z. P. et al. Induction of human neuronal cells by defined transcription factors. Nature 476, 220-223 (2011).

9. Huang, P. et al. Direct reprogramming of human fibroblasts to functional and expandable hepatocytes. Cell Stem Cell 14, 370-384 (2014). 
10. Xu, J., Du, Y. \& Deng, H. Direct lineage reprogramming: strategies, mechanisms, and applications. Cell Stem Cell 16, 119-134 (2015).

11. Si-Tayeb, K., Lemaigre, F. P. \& Duncan, S. A. Organogenesis and development of the liver. Dev. Cell 18, 175-189 (2010).

12. He, Z. et al. Liver xeno-repopulation with human hepatocytes in $\mathrm{Fah}^{-/} \mathrm{Rag}^{-1}$ mice after pharmacological immunosuppression. Am. J. Pathol. 177, 1311-1319 (2010).

13. Yamada, T. et al. In vitro differentiation of embryonic stem cells into hepatocytelike cells identified by cellular uptake of indocyanine green. Stem Cells 20, 146-154 (2002)

14. He, Z. Y. et al. Murine embryonic stem cell-derived hepatocytes correct metabolic liver disease after serial liver repopulation. Int. J. Biochem. Cell. Biol. 44, 648-658 (2012).

15. Guengerich, F. P. Cytochrome P450 enzymes in the generation of commercial products. Nat. Rev. Drug. Discov. 1, 359-366 (2002).

16. Pan, G., Boiselle, C. \& Wang, J. Assessment of biliary clearance in early drug discovery using sandwich-cultured hepatocyte model. J. Pharm. Sci. 101, 1898-1908 (2012).

17. Laursen, L. A preventable cancer. Nature 516, S2-S3 (2014)

18. Sia, D., Villanueva, A., Friedman, S. L. \& Llovet, J. M. Liver Cancer Cell of Origin, Molecular Class, and Effects on Patient Prognosis. Gastroenterology 152, 745-761 (2016).

19. Morise, Z. et al. Recent advances in the surgical treatment of hepatocellular carcinoma. World J. Gastroenterol. 20, 14381-14392 (2014).

20. Costa, R. H., Kalinichenko, V. V., Holterman, A. X. \& Wang, X. Transcription factors in liver development, differentiation, and regeneration. Hepatology $\mathbf{3 8}$, 1331-1347 (2003).

21. Ning, B. F. et al. Hepatocyte nuclear factor 4 alpha suppresses the development of hepatocellular carcinoma. Cancer Res. 70, 7640-7651 (2010).
22. Zeng, X. et al. Recombinant adenovirus carrying the hepatocyte nuclear factor1 alpha gene inhibits hepatocellular carcinoma xenograft growth in mice. Hepatology 54, 2036-2047 (2011).

23. Ning, B. F. et al. Hepatocyte nuclear factor 4alpha-nuclear factor-kappaB feedback circuit modulates liver cancer progression. Hepatology 60, 1607-1619 (2014).

24. Miyoshi, N. et al. Defined factors induce reprogramming of gastrointestinal cancer cells. Proc. Natl Acad. Sci. USA 107, 40-45 (2010).

25. Hochedlinger, K. et al. Reprogramming of a melanoma genome by nuclear transplantation. Genes Dev. 18, 1875-1885 (2004).

26. Lai, J., Kong, C. M., Mahalingam, D., Xie, X. \& Wang, X. Elite model for the generation of induced pluripotent cancer cells (iPCS). PLoS. One. 8, e56702 (2013).

27. Duncan, A. W. et al. Aneuploidy as a mechanism for stress-induced liver adaptation. J. Clin. Invest. 122, 3307-3315 (2012).

28. Duncan, A. W. et al. Frequent aneuploidy among normal human hepatocytes. Gastroenterology 142, 25-28 (2012).

29. Grompe, M. et al. Loss of fumarylacetoacetate hydrolase is responsible for the neonatal hepatic dysfunction phenotype of lethal albino mice. Genes \& Dev. 7, 2298-2307 (1993).

30. Overturf, K. et al. Hepatocytes corrected by gene therapy are selected in vivo in a murine model of hereditary tyrosinaemia type I. Nat. Genet. 12, 266-273 (1996).

31. Azuma, H. et al. Robust expansion of human hepatocytes in $\mathrm{Fah}^{-/-} / \mathrm{Rag}^{-/-} / 112 \mathrm{rg}^{-1}$ mice. Nat. Biotechnol. 25, 903-910 (2007).

32. Kourtidis, A. et al. Distinct E-cadherin-based complexes regulate cell behaviour through miRNA processing or Src and p120 catenin activity. Nat. Cell Biol. 17, 1145-1157 (2015).

33. Gerard, C., Tys, J. \& Lemaigre, F. P. Gene regulatory networks in differentiation and direct reprogramming of hepatic cells. Semin. Cell Dev. Biol. 66, 43-50 (2017) 